\title{
Top quark mass dependence of the Higgs boson-gluon form factor at three loops
}

\author{
Joshua Davies, ${ }^{1}$ Ramona Gröber, ${ }^{2}$ Andreas Maier, ${ }^{3}$ Thomas Rauh, ${ }^{4}$ and Matthias Steinhauser ${ }^{1}$ \\ ${ }^{1}$ Institut für Theoretische Teilchenphysik, Karlsruhe Institute of Technology (KIT), \\ Wolfgang-Gaede Straße 1, 76128 Karlsruhe, Germany \\ ${ }^{2}$ Humboldt-Universität zu Berlin, Institut für Physik, Newtonstraße 15, 12489 Berlin, Germany \\ ${ }^{3}$ Deutsches Elektronen-Synchrotron, DESY, Platanenallee 6, 15738 Zeuthen, Germany \\ ${ }^{4}$ Albert Einstein Center for Fundamental Physics, Institute for Theoretical Physics, \\ University of Bern, Sidlerstrasse 5, CH-3012 Bern, Switzerland
}

(Received 7 June 2019; published 16 August 2019)

\begin{abstract}
We compute three-loop corrections to the Higgs boson-gluon form factor, incorporating the top quark mass dependence. Our method is based on the combination of expansions around the top threshold and for large top quark mass, using conformal mapping and Padé approximation to describe the form factor over the full kinematic range.
\end{abstract}

DOI: 10.1103/PhysRevD.100.034017

\section{INTRODUCTION}

The precise measurement of the properties of the Higgs boson, in particular the coupling strength to other particles and to itself, will be among the main focuses in particle physics in the coming years. The success of this enterprise crucially depends on the accuracy of the predictions provided by the theory community.

A quantity which is available to high perturbative order is the total cross section for the production of a Higgs boson at the Large Hadron Collider (LHC). For a comprehensive collection of relevant works we refer to Ref. [1], but we remark here that QCD corrections including the exact dependence on the top quark mass, $m_{t}$, have been available at next-to-leading order (NLO) for about 25 years [2]. At higher orders only approximate results are available; at next-to-next-to-leading order (NNLO) the infinite top quark mass results from Refs. [3-5] have been complemented by power-suppressed terms in the inverse top quark mass in [6-9]. The $\mathrm{N}^{3} \mathrm{LO}$ result has been obtained in the $m_{t} \rightarrow \infty$ limit in $[10,11]$.

In Ref. [1] several sources of uncertainties have been identified for the prediction of the total cross section. Among them is that of the exact top quark mass dependence of the NNLO corrections which has been estimated to be $1 \%$. In this paper we provide results for the Higgs boson-gluon form factor at three-loop order which constitutes the virtual

Published by the American Physical Society under the terms of the Creative Commons Attribution 4.0 International license. Further distribution of this work must maintain attribution to the author(s) and the published article's title, journal citation, and DOI. Funded by SCOAP ${ }^{3}$. corrections to the production cross section. Thus the findings of this paper help to eliminate the aforementioned uncertainty to a large extent. The Higgs boson-gluon form factor is also an important ingredient for processes where the relevant energy in the fermion loops reaches values close to or above the top quark threshold and the infinite top quark mass limit cannot be applied anymore. This concerns, e.g., Higgs boson pair production via $g g \rightarrow H^{\star} \rightarrow H H$ or the measurement of the Higgs boson width from off-shell production of $Z$ boson pairs in gluon fusion via $g g \rightarrow H^{\star} \rightarrow Z Z$ [12]. The exact dependence on the fermion mass in the loop is also important for numerous theories beyond the Standard Model, which often contain additional heavier Higgs bosons.

At two-loop order exact results for the form factor are known from Refs. [2,13-15]. However, at three loops only expansions for large top quark mass $[16,17]$ and nonanalytic terms in the expansion around the top threshold up to $\mathcal{O}(1-z)[18]$ are known, where

$$
z=\frac{\hat{s}}{4 m_{t}^{2}}
$$

with $\sqrt{\hat{s}}$ being the partonic center-of-mass energy. For later convenience we also introduce $\bar{z}=1-z$. In the next section we describe our method which we use to combine these expansions in order to obtain results for the form factor valid for all spacelike and timelike momentum transfers. In Sec. III we discuss our results and Sec. IV contains a brief summary.

\section{METHOD}

The method we use for the construction of the top quark mass dependence of the Higgs boson-gluon form factor is 

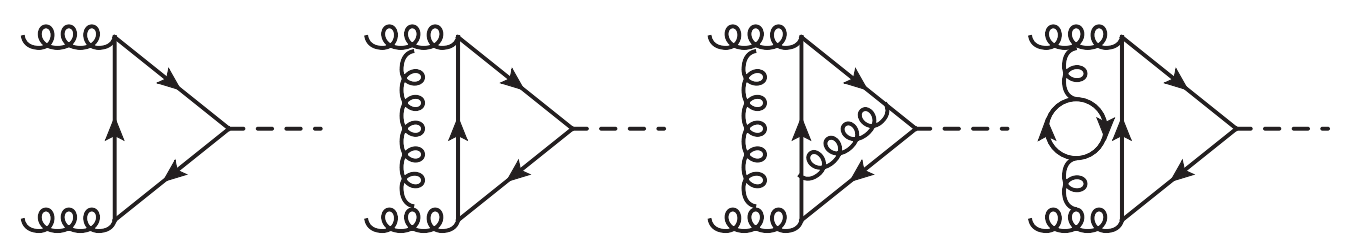

FIG. 1. One-, two- and three-loop Feynman diagrams contributing to $F_{\triangle}$. Solid, curly and dashed lines represent quarks, gluons and Higgs bosons, respectively.

based on the efficient combination of information from the large top quark mass expansion (LME) $(z \rightarrow 0)$ and knowledge from the threshold where $\hat{s} \approx 4 m_{t}^{2}(z \rightarrow 1)$, using conformal mapping and Padé approximation. The procedure was developed in Ref. [19] (see also [20,21]) in order to compute a certain class of four-loop contributions to the muon anomalous magnetic moment. In Refs. [22,23] the method was extended to QCD corrections with the aim to compute NNLO correction to the total cross section $\sigma\left(e^{+} e^{-} \rightarrow\right.$ hadrons $)$. A further refinement of the method has been developed in Refs. [24,25] where order $\alpha_{s}^{3}$ corrections to $\sigma\left(e^{+} e^{-} \rightarrow\right.$ hadrons $)$ have been computed. In these references additional parameters were introduced which allow one to generate a larger number of Padé approximations and thus obtain more reliable uncertainty estimates. The systematic improvement of the Padé approximations when increasing the number of input terms has been studied in Ref. [26]. In Ref. [18] the method has been used to obtain two-loop corrections for the three form factors relevant for Higgs boson pair production.

In the following we briefly describe the application of this method to the form factor entering the interaction of a Higgs boson and two gluons. We parametrize the corresponding amplitude as

$$
\begin{aligned}
& \mathcal{A}_{a b}^{\mu \nu}(g g \rightarrow H) \\
& \quad=\delta_{a b} \frac{y_{t}}{2 \sqrt{2} m_{t}} \frac{\alpha_{s}}{\pi} T_{F}\left(q_{1} \cdot q_{2} g^{\mu \nu}-q_{1}^{\nu} q_{2}^{\mu}\right) F_{\triangle}(z),
\end{aligned}
$$

where $q_{1}$ and $q_{2}$ are the external momenta of the gluons with polarization vectors $\varepsilon^{\mu}\left(q_{1}\right)$ and $\varepsilon^{\nu}\left(q_{2}\right)$, respectively. $y_{t}=\sqrt{2} m_{t} / v$ is the top quark Yukawa coupling, $v$ is the vacuum expectation value, $a$ and $b$ are adjoint color indices, $T_{F}=1 / 2$ and $\hat{s}=\left(q_{1}+q_{2}\right)^{2}=2 q_{1} \cdot q_{2}$. It is convenient to define the perturbative expansion of $F_{\triangle}$ as

$$
F_{\triangle}=F_{\triangle}^{(0)}+\frac{\alpha_{s}}{\pi} F_{\triangle}^{(1)}+\left(\frac{\alpha_{s}}{\pi}\right)^{2} F_{\triangle}^{(2)}+\cdots,
$$

where $\alpha_{s} \equiv \alpha_{s}^{(5)}(\mu)$ is the strong coupling constant with five active flavors evaluated at the renormalization scale $\mu$. Sample Feynman diagrams contributing to $\mathcal{A}_{a b}^{\mu \nu}(g g \rightarrow H)$ up to three loops can be found in Fig. 1.

The one-loop result, $F_{\triangle}^{(0)}$, is finite. At two-loop order we renormalize the gluon wave function and the top quark mass in the on-shell scheme and the strong coupling constant in the $\overline{\mathrm{MS}}$ scheme. Note that the ultraviolet renormalized form factor still contains infrared divergences which cancel against contributions from real radiation, in order to form finite physical quantities. The structure of the infrared divergences is universal and has been studied in detail in the literature [27]. In our case finite form factors are obtained via

$$
\begin{aligned}
& F_{\triangle}^{(1) \text {,fin }}=F_{\triangle}^{(1)}-\frac{1}{2} I_{g}^{(1)} F_{\triangle}^{(0)}, \\
& F_{\triangle}^{(2) \text {,fin }}=F_{\triangle}^{(2)}-\frac{1}{2} I_{g}^{(1)} F_{\triangle}^{(1)}-\frac{1}{4} I_{g}^{(2)} F_{\triangle}^{(0)},
\end{aligned}
$$

where $I_{g}^{(1)}$ and $I_{g}^{(2)}$ can be found in Refs. [27,28]. In order to fix the notation we provide an explicit expression only for $I_{g}^{(1)}$ which is given by

$$
I_{g}^{(1)}=-\left(\frac{\mu^{2}}{-\hat{s}-i \delta}\right)^{\epsilon} \frac{e^{\epsilon \gamma_{E}}}{\Gamma(1-\epsilon)} \frac{1}{\epsilon^{2}}\left[C_{A}+2 \epsilon \beta_{0}\right],
$$

with $\beta_{0}=\left(11 C_{A}-4 T_{F} n_{l}\right) / 12$, where $C_{A}=3, T_{F}=1 / 2$ and $n_{l}$ is the number of massless quarks. We work in $d=$ $4-2 \epsilon$ dimensions and assume that $\delta$ is an infinitesimal small parameter. We apply the method described below to $F_{\triangle}^{(1), \text { fin }}$ and $F_{\triangle}^{(2), \text { fin }}$.

In the following we briefly discuss the input for the limits $z \rightarrow 0$ and $z \rightarrow 1$ used for the construction of the Padé approximants. For the renormalization scale we choose $\mu^{2}=-\hat{s}$ since the $\mu$ dependence can easily be reconstructed from the one- and two-loop expressions, which are known exactly, see Ref. [29]. Furthermore, we set all color factors to their numerical values and only keep $n_{l}$ as a parameter. The large- $m_{t}$ expansion of the three-loop form factor up to order $z^{4}$ has been computed in Refs. $[16,17]$ and the $z^{5}$ and $z^{6}$ terms are available from Ref. [29]. The analytic expressions read 


$$
\begin{aligned}
& F_{\triangle}^{(0)}=\frac{4}{3}+\frac{14}{45} z+\frac{8}{63} z^{2}+\frac{104}{1575} z^{3}+\frac{2048}{51975} z^{4}+\frac{4864}{189189} z^{5}+\frac{512}{28665} z^{6}+\mathcal{O}\left(z^{7}\right), \\
& F_{\triangle}^{(1), \text { fin }}=\frac{11}{3}+\frac{1237}{810} z+\frac{35726}{42525} z^{2}+\frac{157483}{297675} z^{3}+\frac{2546776}{7016625} z^{4}+\frac{194849538824}{737482370625} z^{5}+\frac{385088204192}{1917454163625} z^{6}+\mathcal{O}\left(z^{7}\right), \\
& F_{\triangle}^{(2), \text { fin }}=-\frac{253 \zeta(3)}{24}+\frac{3941}{108}+\frac{19 \pi^{2}}{12}+\frac{\pi^{4}}{96}+\frac{19}{12} L_{s}+n_{l}\left(-\frac{17 \zeta(3)}{36}-\frac{3239}{648}-\frac{47 \pi^{2}}{432}+\frac{4}{9} L_{s}\right)+n_{l}^{2} \frac{\pi^{2}}{648} \\
& +\left[\frac{9290881 \zeta(3)}{103680}-\frac{44326367}{466560}+\frac{623 \pi^{2}}{1080}+\frac{7 \pi^{4}}{2880}+\frac{28}{405} \pi^{2} \log (2)+\frac{8261}{3240} L_{s}\right. \\
& \left.+n_{l}\left(-\frac{119 \zeta(3)}{1080}-\frac{107087}{291600}-\frac{259 \pi^{2}}{4320}-\frac{169}{1080} L_{s}\right)+n_{l}^{2} \frac{7 \pi^{2}}{19440}\right] z \\
& +\left[\frac{7037623781 \zeta(3)}{69672960}-\frac{82500975779}{731566080}+\frac{121 \pi^{2}}{378}+\frac{\pi^{4}}{1008}+\frac{32}{567} \pi^{2} \log (2)\right. \\
& \left.+\frac{253549}{170100} L_{s}+n_{l}\left(-\frac{17 \zeta(3)}{378}-\frac{6385481}{53581500}-\frac{25 \pi^{2}}{648}-\frac{4133}{36450} L_{s}\right)+n_{l}^{2} \frac{\pi^{2}}{6804}\right] z^{2} \\
& +\left[\frac{650760513719 \zeta(3)}{412876800}-\frac{1740869750908152049}{921773260800000}+\frac{221 \pi^{2}}{1050}+\frac{13 \pi^{4}}{25200}+\frac{208 \pi^{2} \log (2)}{4725}+\frac{804644}{826875} L_{s}\right. \\
& \left.+n_{l}\left(-\frac{221 \zeta(3)}{9450}-\frac{6383750249}{112521150000}-\frac{3107 \pi^{2}}{113400}-\frac{1147037}{14883750} L_{s}\right)+n_{l}^{2} \frac{13 \pi^{2}}{170100}\right] z^{3} \\
& +\left[\frac{193543938976537 \zeta(3)}{37158912000}-\frac{6978205934887756008911}{1115345645568000000}+\frac{4736 \pi^{2}}{31185}+\frac{16 \pi^{4}}{51975}\right. \\
& \left.+\frac{16384 \pi^{2} \log (2)}{467775}+\frac{33498106}{49116375} L_{s}+n_{l}\left(-\frac{2176 \zeta(3)}{155925}-\frac{2197298833}{72937816875}-\frac{3232 \pi^{2}}{155925}-\frac{20932}{382725} L_{s}\right)+n_{l}^{2} \frac{64 \pi^{2}}{1403325}\right] z^{4} \\
& +\left[\frac{2460310706266276921 \zeta(3)}{81155063808000}-\frac{159929147625953730170902566067}{4389031448658778521600000}\right. \\
& +\frac{9424 \pi^{2}}{81081}+\frac{38 \pi^{4}}{189189}+\frac{48640 \pi^{2} \log (2)}{1702701}+\frac{945911804923}{1877227852500} L_{s} \\
& \left.+n_{l}\left(-\frac{5168 \zeta(3)}{567567}-\frac{22552503119716043}{1395235522161731250}-\frac{27892 \pi^{2}}{1702701}-\frac{48324340168}{1191317675625} L_{s}\right)+n_{l}^{2} \frac{152 \pi^{2}}{5108103}\right] z^{5} \\
& +\left[\frac{15128773883548934558969 \zeta(3)}{114266329841664000}+\frac{2656 \pi^{2}}{28665}+\frac{4 \pi^{4}}{28665}+\frac{2048 \pi^{2} \log (2)}{85995}\right. \\
& -\frac{13560383230749413568271118392175429}{85205730523295753699328000000}+\frac{339242844181}{871570074375} L_{s} \\
& \left.+n_{l}\left(-\frac{544 \zeta(3)}{85995}-\frac{2085146760850288}{259115168401464375}-\frac{3448 \pi^{2}}{257985}-\frac{35895528824}{1150472498175} L_{s}\right)+n_{l}^{2} \frac{16 \pi^{2}}{773955}\right] z^{6}+\mathcal{O}\left(z^{7}\right),
\end{aligned}
$$

where $L_{s}=\log (-4 z-i 0)$ and $\zeta(n)$ is the Riemann zeta function.

The expansion of the three-loop form factor around the threshold was considered in Ref. [18] in the effective theory of nonrelativistic QCD (NRQCD). We briefly outline the approach and refer to [18] for details.

Within NRQCD, the leading contributions to the triangle form factor near the threshold can be written schematically as

$$
F_{\triangle}(z) \stackrel{z \rightarrow^{1}}{=} C_{g g \rightarrow t \bar{t}} C_{t \bar{t} \rightarrow H} G_{P}(z)
$$

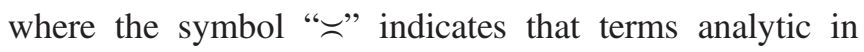
$(1-z)$ have been dropped on the right-hand side. The relativistic short-distance corrections to top pair production and annihilation are absorbed into the matching coefficients $C_{g g \rightarrow t \bar{t}}, C_{t \bar{t} \rightarrow H}$ and the propagation of the intermediate nonrelativistic $t \bar{t}$ pair is described by the Coulomb resummed $P$-wave Green function $G_{P}(z)$ [30]. 
Expanding Eq. (7) in $\alpha_{s}$ yields the perturbative coefficients of the form factor. An explicit result for the three-loop form factor is given in Eq. (50) of [18]. For convenience we reproduce the analytic expression together with the oneand two-loop results which are given by

$$
\begin{aligned}
& F_{\triangle}^{(0)} \stackrel{z \rightarrow 1}{\asymp} 2 \pi(1-z)^{3 / 2}+\frac{13 \pi}{3}(1-z)^{5 / 2}+\mathcal{O}\left((1-z)^{7 / 2}\right), \\
& F_{\triangle}^{(1), \text { fin } z \rightarrow \underbrace{\prime}} \frac{4 \pi^{2}}{3}(1-z) \log (1-z)-\frac{\pi}{36}\left(124+15 \pi^{2}\right)(1-z)^{3 / 2} \\
& +\frac{8 \pi^{2}}{9}(1-z)^{2} \log (1-z)+\frac{\pi}{216}\left[2252-117 \pi^{2}\right. \\
& -2112 \log (2)-672 \log (1-z)](1-z)^{5 / 2} \\
& -\frac{28 \pi^{2}}{45}(1-z)^{3} \log (1-z)+\mathcal{O}\left((1-z)^{7 / 2}\right),
\end{aligned}
$$

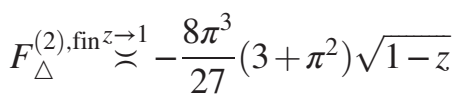

$$
\begin{aligned}
& +\frac{\pi^{2}}{54}\left[\left(458-15 \pi^{2}-44 n_{l}\right) \log (1-z)\right. \\
& \left.-\left(99-6 n_{l}\right) \log ^{2}(1-z)\right](1-z)+\mathcal{O}\left((1-z)^{3 / 2}\right) \text {. }
\end{aligned}
$$

The information provided in Eqs. (6) and (8) is used to construct approximations of the form factor. We first subtract the logarithmic contributions for $z \sim 1$ and define

$$
\tilde{F}_{\triangle}=F_{\triangle}-F_{\triangle}^{\text {sub }},
$$

where $F_{\triangle}^{\text {sub }}$ is constructed to both be analytic for $|z|<1$ and to reproduce the threshold logarithms in Eq. (8), so that the threshold expansion of $\tilde{F}_{\triangle}$ is free of logarithms up to $(1-z)^{3 / 2}$. Such a subtraction function $F_{\triangle}^{\text {sub }}$ can be obtained using the vacuum polarization as a building block, see [18] for details of the construction. For explicit examples for $F_{\triangle}^{\text {sub }}$ we refer to the sample Padé approximants in the Supplemental Material [31]. Note that also in the limit $z \rightarrow 0 F_{\triangle}$ develops logarithmic divergences which manifest in the linear $L_{s}$ term in Eq. (6). Whereas in Ref. [23] these contributions are also subtracted, here we instead construct separate Padé approximants for the $L_{s}$-independent term and for the coefficient of $L_{s}$, as discussed in [18].

Next we apply a conformal mapping,

$$
z=\frac{4 \omega}{(1+\omega)^{2}},
$$

to transform the $z$ plane into the interior of the unit circle in the $\omega$ plane; the timelike momentum regions $z \in[0,1]$ and $z \in[1, \infty]$ with $\operatorname{Im}(z)>0$ are mapped to $\omega \in[0,1]$ and the upper semicircle, respectively.

At this point we construct Padé approximants in the variable $\omega$. They have the form

$$
[n / m](\omega)=\frac{\sum_{i=0}^{n} a_{i} \omega^{i}}{1+\sum_{j=1}^{m} b_{j} \omega^{j}},
$$

where $n+m$ is fixed by the number of input terms from the large top mass and threshold expansions. In our case we have seven terms for $z \rightarrow 0$ and one for $z \rightarrow 1$ which is sufficient to determine eight coefficients in Eq. (11), i.e., Padé approximants for $n+m=7$. More precisely, we construct Padé approximants for the rescaled form factor

$$
[n / m](\omega) \simeq\left[1+a_{R} z(\omega)\right] \tilde{F}_{\triangle}(z(\omega)),
$$

where $a_{R}$ is a real parameter. This removes the spurious condition $F_{\triangle}(z \rightarrow \infty)=0$ introduced by the definition of the form factor through $\mathcal{A}_{g g \rightarrow H} \propto z F_{\triangle}(z)$ and provides a means to test the stability of the solutions through variation of $a_{R}$. As discussed in [18] we only use the diagonal and next-to-diagonal Padé approximants which are $[5 / 2],[4 / 3],[3 / 4]$ and $[2 / 5]$ in the case that seven large top quark mass expansion terms and one term from the threshold expansion are taken into account. In Sec. III we also show results which only incorporate LME terms up to $z^{4}$, for which we construct the Padé approximants [4/1], [3/2], [2/3] and [1/4].

By construction the Padé approximants develop poles in the complex $\omega$ plane. In the following we discuss our criteria which exclude approximants with poles too close to the physical region. For this discussion we have to distinguish spacelike and timelike momentum regions. For $z>0$ we exclude all approximants which contain poles $\omega_{0}$ in the region

$$
\operatorname{Re}\left(z\left(\omega_{0}\right)\right) \geq-2 \quad \text { and } \quad\left|\omega_{0}\right| \leq 1.2,
$$

as they can cause unphysical behavior in the approximation. We find that poles in the entire complex plane in $z$, i.e., in the unit disc $|\omega| \leq 1$, cannot be excluded as this leads to the exclusion of all Padé approximants. In those cases where the approximants show obviously unphysical resonances we moderately increase the exclusion region. This concerns the coefficient of $L_{s}$ for $F_{\triangle}^{(2,0), f i n}(z)$ [cf. Eq. (17)] where we use

$$
\operatorname{Re}\left(z\left(\omega_{0}\right)\right) \geq-2 \quad \text { and } \quad\left|\omega_{0}\right| \leq 1.3 .
$$

For each choice of $[n / m]$ we aim to construct 20 Padé approximants by choosing $a_{R}$ in Eq. (12) randomly in the range $[0.1,10]$, leading to a maximum of 80 approximants. The mean and standard deviation of this set are used as the central value and uncertainty estimate, respectively. For some choices of $\{n, m\}$ Padé approximants satisfying criteria (13) and (14) could not be found, however, we checked that at least 40 approximants remain in all cases. For such sets of fewer than 80 approximants we increase our uncertainty estimate by the ratio of the maximal 


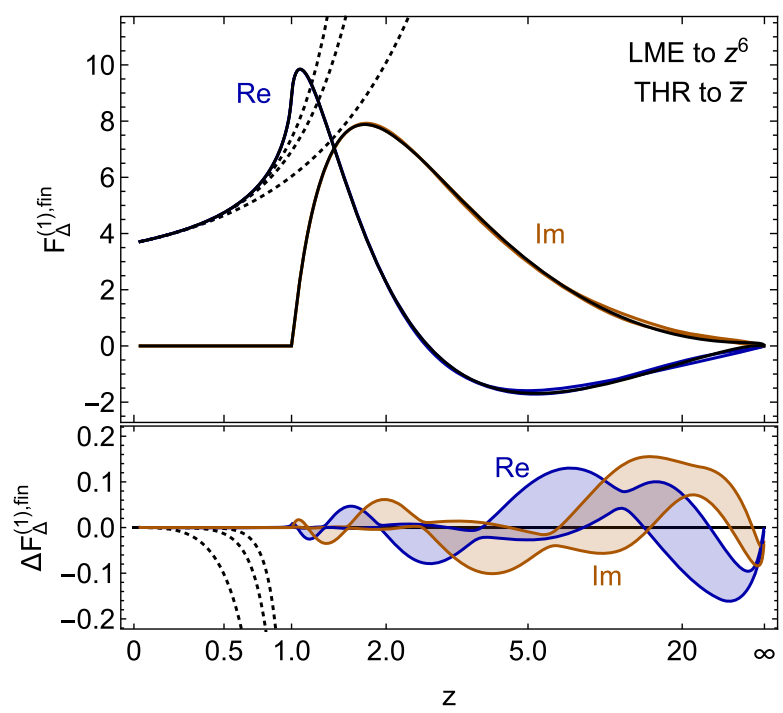

FIG. 2. The upper panel shows our approximations for the real and imaginary parts of the two-loop triangle form factor in blue and orange, respectively. The bands give the standard deviation of the Padé approximants which we consider. The exact results are shown in black. The dashed lines correspond to the real part of the LME approximation up to order $z^{2}, z^{4}$ and $z^{6}$. The lower panel shows the difference between the exact result and the approximations.

number of Padé approximants (80) over the actual number in the set.

For spacelike momenta our exclusion region is defined by

$$
\operatorname{Re}\left(z\left(\omega_{0}\right)\right) \leq 2 \text { and }\left|\omega_{0}\right| \leq 1.2
$$

and negative values of $a_{R}$ in the range $[-10,-0.1]$ are chosen.

\section{RESULTS}

Before discussing the three-loop results we apply the method described in the previous section to the two-loop form factor, for which we can compare to the exact expressions [2,13-15].

We show in Fig. 2 that the exact result for the two-loop form factor can be reproduced very well with the same amount of information that is available at three loops. The shaded region is spanned by the standard deviation with respect to the mean value of a set of 20 approximants for each considered set $\{n, m\}$. These approximants are available in the Supplemental Material [31]. Figure 3, where the difference between the exact result and the approximations is shown, demonstrates that the approximation can be systematically improved by including more expansion coefficients. We compare the results based on the input used in Fig. 2 (lower left panel) to results where fewer expansion coefficients for large top quark masses are used (upper left panel). Furthermore, we also show results where additional information from the threshold is incorporated in the construction of the Padé approximations (panels on the right).

Our approximation of the three-loop form factor is shown in Fig. 4 and represents the main result of this paper. At three loops the LME and threshold coefficients

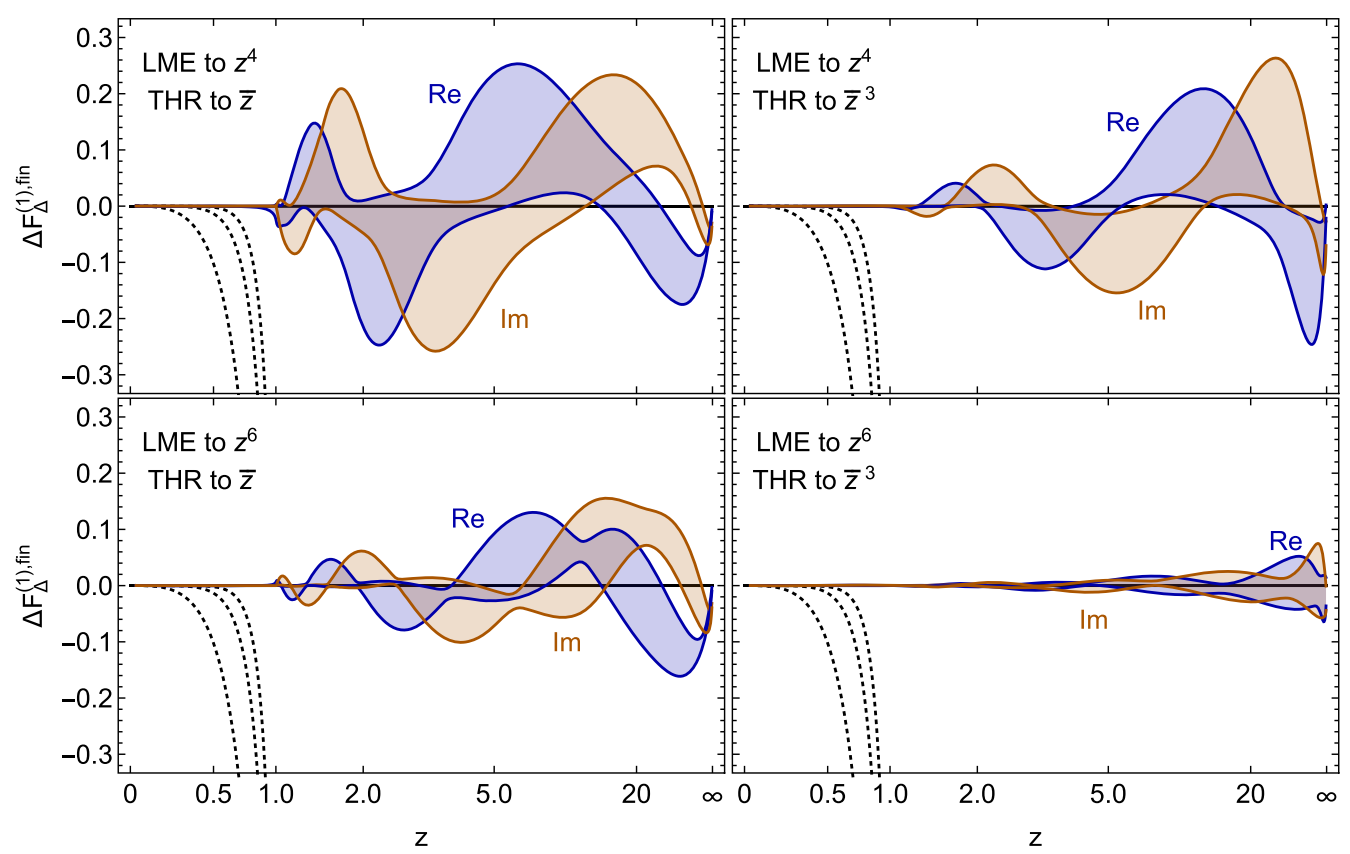

FIG. 3. Differences between our approximations and the exact result. The input used is shown in the legend of each panel. The dashed lines correspond to the real part of the LME approximation up to order $z^{2}, z^{4}$ and $z^{6}$. The lower left panel corresponds to the lower panel of Fig. 2. 


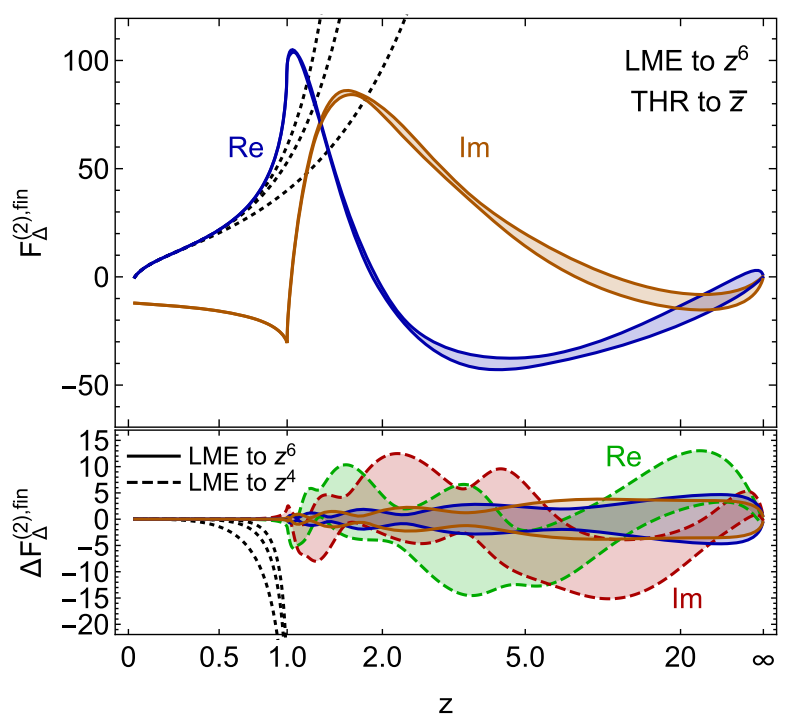

FIG. 4. Our approximations for the real and imaginary parts of the three-loop triangle form factor are shown in blue and orange, respectively. The bands give the standard deviation of the considered set of Padé approximants. The dashed lines correspond to the real part up to order $z^{2}, z^{4}$ and $z^{6}$. The lower panel shows the differences to the central values; see text for details.

develop terms linear in $L_{s}=\log (-4 z-i 0)$. We construct separate Padé approximants for the coefficient such that we obtain an approximation of the form

$F_{\triangle}^{(2), \text { fin }}(z(\omega)) \simeq \frac{[n / m]_{0}(\omega)+F_{\triangle}^{(2), \text { sub }}}{1+a_{R, 0} z(\omega)}+\frac{[k / l]_{1}(\omega) L_{s}}{1+a_{R, 1} z(\omega)}$,

where the subscripts indicate the power of $L_{s}$. Note that the Padé approximants of the $L_{s}$-independent and linear- $L_{s}$ term are averaged independently using separate values of
$a_{R}$. The threshold subtraction [cf. Eq. (9)] is only needed for the first term in Eq. (16). The lower panel in Fig. 4 shows the differences from the central values (obtained using seven expansion terms for small $z$ ) both with seven and five input terms from the large top quark mass expansion as solid and dashed boundaries of the uncertainty bands, respectively. One observes over the whole range in $z$ (except for a small region for $z \approx 10$ ) that the solid bands lie within the dashed band. Below threshold $(z=1)$ our method results in tiny uncertainties for both the real and imaginary parts of the form factor. For $1 \leq z \leq 2$ the form factor is numerically large and we thus observe small relative uncertainties. Although the absolute uncertainty becomes larger for higher values of $z$ we can provide a good approximation with an uncertainty which is sufficiently small for phenomenological applications.

In order to facilitate the comparison with a future exact calculation we split our three-loop result according to the number of light fermions and write

$F_{\triangle}^{(2), \text { fin }}(z)=F_{\triangle}^{(2,0), \text { fin }}(z)+n_{l} F_{\triangle}^{(2,1), \text { fin }}(z)+n_{l}^{2} F_{\triangle}^{(2,2), \text { fin }}(z)$,

where $n_{l}=5$ is the number of light flavors. Note that $F_{\triangle}^{(2,0) \text {,fin }}(z)$ contains contributions with closed massive loops, which are numerically less important than the $n_{l}$ terms. There are no three-loop vertex diagrams which contain two closed fermion loops; $F_{\triangle}^{(2,2) \text {,fin }}(z)$ is completely determined by the infrared subtraction terms. In fact, it is proportional to $F_{\triangle}^{(0)}$ and we will not discuss it further.

The results for $F_{\triangle}^{(2,0) \text {,fin }}(z)$ and $F_{\triangle}^{(2,1) \text {,fin }}(z)$ are shown in Fig. 5, adopting the notation from Fig. 4. Both coefficients show a convergence which is very similar to $F_{\triangle}^{(2) \text {,fin }}$. Summing up the coefficients leads to good agreement with
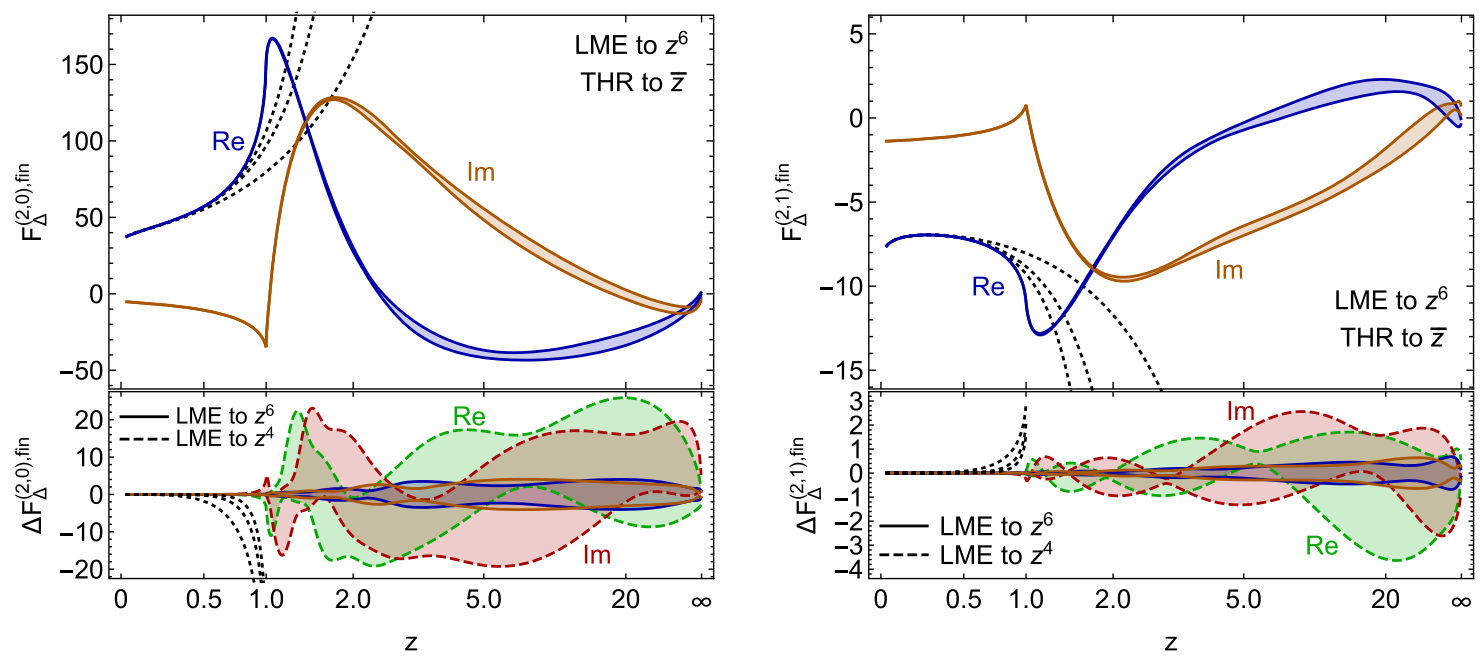

FIG. 5. Our approximations for the three-loop form factor separated according to the light-fermion contributions. 


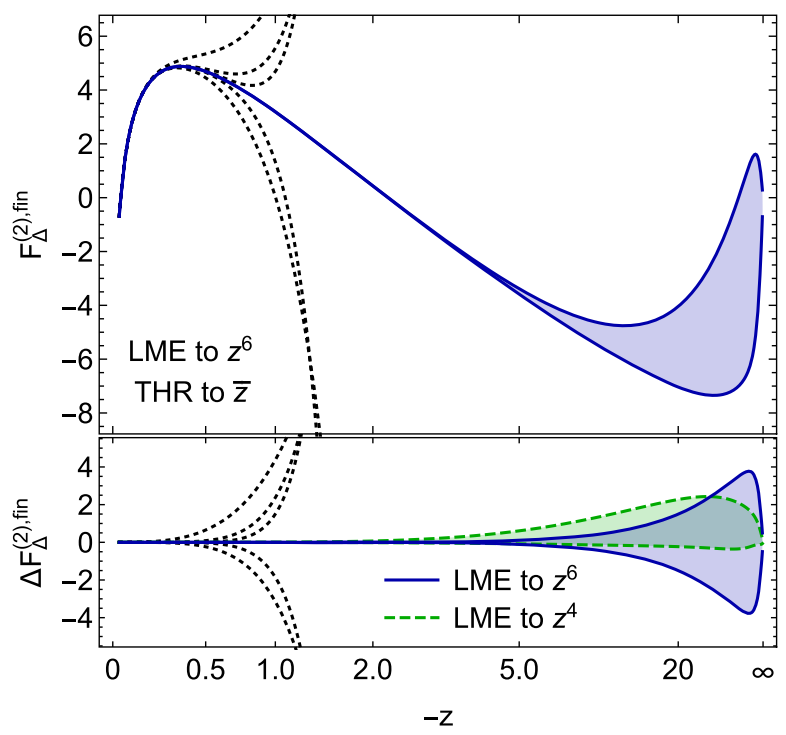

FIG. 6. The three-loop result for the form factor $F_{\triangle}^{(2), \text { fin }}$ for spacelike momenta. The dashed lines correspond to the real part of the LME approximation up to order $z^{2}, z^{3}, z^{4}, z^{5}$ and $z^{6}$. The same notation as in Fig. 5 is adopted.

the result (16) but with a larger uncertainty which is why (16) should be used for numerical applications. Note that $F_{\triangle}^{(2,0) \text {,fin }}(z)$ is the only result where the exclusion criterion (14) has been used whereas for all other results (13) is applied.

Finally, we present in Fig. 6 results for the three-loop form factor for $z<0$. One observes small uncertainties for $|z|<5$ which become larger when $z$ becomes more negative. For $|z|>20$ the Padé approximation procedure does not lead to stable results which is also seen by the fact that the uncertainty becomes larger after incorporating more expansion terms (see lower panel). Note that the large top quark mass expansion shows an alternating behavior.
Together with this paper we provide representative Padé approximants for all plots shown in this section in Supplemental Material [31].

\section{CONCLUSION}

We compute three-loop corrections to the Higgs bosongluon form factor including finite top quark mass effects. Our approach is based on the combination of analytic results from two kinematic regions: the expansion for large top quark mass and the top quark threshold. In addition, we incorporate the information that the form factors vanish at high energies by a rescaling [cf. Eq. (12)]. For the rescaled form factors, we apply a conformal mapping and a subsequent Padé approximation. We first apply our method at two loops and show that we can reproduce the known results. The two-loop expression is also used to demonstrate that our estimate for the uncertainty works reliably. Our main result is shown in Fig. 4 where we plot the threeloop form factor in the timelike momentum region. This plot can be reproduced using the approximation functions which are provided in the Supplemental Material [31]. We have shown that our results can be systematically improved by incorporating more expansion terms into the analysis.

\section{ACKNOWLEDGMENTS}

R. G. is supported by the "Berliner Chancengleichheitsprogramm." This research was supported by the Deutsche Forschungsgemeinschaft (DFG, German Research Foundation) under Grant No. 396021762-TRR 257 "Particle Physics Phenomenology after the Higgs Discovery" and has received funding from the European Union's Horizon 2020 research and innovation program under the Marie Skłodowska-Curie Grant Agreement No. 764850, SAGEX. We thank Robert Harlander, Mario Prausa and Johann Usovitsch for pointing out a missing factor $1 / 2$ in Eq. (2) in the first version of the manuscript.
[1] D. de Florian et al. (LHC Higgs Cross Section Working Group), arXiv:1610.07922.

[2] M. Spira, A. Djouadi, D. Graudenz, and P. M. Zerwas, Nucl. Phys. B453, 17 (1995).

[3] R. V. Harlander and W. B. Kilgore, Phys. Rev. Lett. 88, 201801 (2002).

[4] C. Anastasiou and K. Melnikov, Nucl. Phys. B646, 220 (2002).

[5] V. Ravindran, J. Smith, and W. L. van Neerven, Nucl. Phys. B665, 325 (2003).

[6] R. V. Harlander and K. J. Ozeren, J. High Energy Phys. 11 (2009) 088.
[7] A. Pak, M. Rogal, and M. Steinhauser, J. High Energy Phys. 02 (2010) 025.

[8] R. V. Harlander, H. Mantler, S. Marzani, and K. J. Ozeren, Eur. Phys. J. C 66, 359 (2010).

[9] A. Pak, M. Rogal, and M. Steinhauser, J. High Energy Phys. 09 (2011) 088.

[10] C. Anastasiou, C. Duhr, F. Dulat, E. Furlan, T. Gehrmann, F. Herzog, A. Lazopoulos, and B. Mistlberger, J. High Energy Phys. 05 (2016) 058.

[11] B. Mistlberger, J. High Energy Phys. 05 (2018) 028.

[12] F. Caola and K. Melnikov, Phys. Rev. D 88, 054024 (2013). 
[13] R. Harlander and P. Kant, J. High Energy Phys. 12 (2005) 015.

[14] C. Anastasiou, S. Beerli, S. Bucherer, A. Daleo, and Z. Kunszt, J. High Energy Phys. 01 (2007) 082.

[15] U. Aglietti, R. Bonciani, G. Degrassi, and A. Vicini, J. High Energy Phys. 01 (2007) 021.

[16] R. V. Harlander and K. J. Ozeren, Phys. Lett. B 679, 467 (2009).

[17] A. Pak, M. Rogal, and M. Steinhauser, Phys. Lett. B 679, 473 (2009).

[18] R. Gröber, A. Maier, and T. Rauh, J. High Energy Phys. 03 (2018) 020.

[19] P. A. Baikov and D. J. Broadhurst, in New Computing Techniques in Physics Research, Pisa 1995, pp. 167-172.

[20] D. J. Broadhurst, J. Fleischer, and O. V. Tarasov, Z. Phys. C 60, 287 (1993).

[21] J. Fleischer and O. V. Tarasov, Z. Phys. C 64, 413 (1994).

[22] K. G. Chetyrkin, J. H. Kühn, and M. Steinhauser, Phys. Lett. B 371, 93 (1996).
[23] K. G. Chetyrkin, R. Harlander, and M. Steinhauser, Phys. Rev. D 58, 014012 (1998).

[24] A. H. Hoang, V. Mateu, and S. Mohammad Zebarjad, Nucl. Phys. B813, 349 (2009).

[25] Y. Kiyo, A. Maier, P. Maierhöfer, and P. Marquard, Nucl. Phys. B823, 269 (2009).

[26] A. Maier and P. Marquard, Phys. Rev. D 97, 056016 (2018).

[27] S. Catani, Phys. Lett. B 427, 161 (1998).

[28] D. de Florian and J. Mazzitelli, J. High Energy Phys. 12 (2012) 088.

[29] J. Davies and M. Steinhauser, Three-loop form factors for Higgs boson pair production in the large top mass limit (to be published).

[30] M. Beneke, J. Piclum, and T. Rauh, Nucl. Phys. B880, 414 (2014).

[31] See Supplemental Material at http://link.aps.org/supplemental/ 10.1103/PhysRevD.100.034017, for computer-readable expressions of the Padé approximants which have been constructed as described in the paper. 